\title{
Occlusal Plane Analyzer: A Customized Device for Determining the Occlusal Plane
}

\author{
Rubina Gupta \\ Assistant Professor, Department of Dental Surgery, Muzaffarnagar Medical College, Muzaffarnagar, Uttar Pradesh, India
}

\begin{abstract}
Correspondence: Rubina Gupta, Assistant Professor, Department of Dental Surgery, Muzaffarnagar Medical College Muzaffarnagar, Uttar Pradesh, India, e-mail: dr.guptarubina@gmail.com

\section{ABSTRACT}

Since times immemorial, dentists have faced the challenge of providing accord between function and esthetics. The occlusal plane, lost in patients rendered edentulous, should be relocated if complete dentures are to be esthetically and functionally satisfactory.

Changes in the plane of occlusion modify the physical and functional relationship of the oral musculature leading to an alteration in function, comfort and also the esthetic value.

Considering the importance of the accurate establishment of the location and the effect of the inclination of the established occlusal plane on function, esthetics and speech, a method to conform it to the occlusal plane that existed in the natural teeth seems necessary.

This paper describes a custom-made instrument to check for the parallelism of ala-tragus line to the occlusal plane and also to check for the parallelism of occlusal plane to interpupillary line.

Keywords: Occlusal plane, Campers plane, Interpupillary line, Complete dentures.
\end{abstract}

\section{INTRODUCTION}

Dentists have always faced the challenge of providing accord between function and esthetics. It can be said that for the success of complete denture prosthesis, arranging the teeth in correct plane of occlusion is pivotal. The orientation of the occlusal plane forms the basis for teeth arrangement conducive to satisfactory esthetics and proper function.

The glossary of prosthodontic terms (July 2005) ${ }^{1}$ defines occlusal plane as 'the average plane established by the incisal and occlusal surfaces of the teeth'. Generally, it is not a plane but represents the planar mean of the curvature of these surfaces. A plane is determined by at least three reference points that are not in a straight line.

Anteriorly, occlusal plane helps in achieving esthetics and phonetics while posteriorly, it forms a milling surface. Functionally, the inclination of the occlusal plane is one of the key factors governing occlusal balance. Changes in the plane of occlusion will modify the physical and functional relationship of the oral musculature leading to an alteration in function, comfort and also the esthetic value.

Hence, the occlusal plane, lost in patients rendered edentulous, should be relocated if complete dentures are to be esthetically and functionally satisfactory.

\section{REVIEW OF LITERATURE}

Numerous authors, since the beginning of complete denture fabrication, have proposed several landmarks to help define the level of occlusal plane. Various planes like Frankfurt horizontal plane, Camper's line, palatal line, occlusal line, mandibular line, interpupillary line, hamular notch incisive papilla (HIP plane), etc. have been suggested. The most commonly used is Camper's plane as suggested by Landa ${ }^{2}$ in 1947, H Okane ${ }^{3}$ in 1979, Arthur O Rahn and Charles M Heartwell $^{4}$ in 1986, F Rostamkhani, A Sahafian and $\mathrm{H}$ Kermani $^{5}$ in 2005, etc.

A number of instruments have also been described in literature for orientation of the occlusal plane. JE Scott in $1952^{6}$ described an instrument 'the bite plane leveler' consisting of two flat, horizontally curved, parallel bars. The instrument can be used to check the parallelism of occlusal plane to the alatragus line only.

Nikzad S Javid (1974) ${ }^{7}$ suggested the use of 'J-plane' with a fox plane to establish the occlusal plane. The J-plane was meant to be used instead of metallic scale. The procedure had all the disadvantages of using fox plane.

Kazanoglu and John W Unger (1992) ${ }^{8}$ described 'Camper's plane indicator' for occlusal plane orientation. The device consisted of two metal plates connected with a long aluminum rod. The orientation with interpupillary line and right and left Camper's plane was done separately making it a time-consuming procedure.

Urbano, Santana-Penin and Maria J Mora $(1998)^{9}$ described another device for determining the inclination of the occlusal plane. The device, made of stainless steel, is U-shaped with one shorter, inner arm designed to be positioned against the occlusal surface and one longer, outer arm that lies outside the mouth. The disadvantage was that a metallic scale was required to check the parallelism. Also, the parallelism with interpupillary line cannot be checked using this device. 


\section{INSTRUMENT (OCCLUSAL PLANE ANALYZER)}

The custom-made instrument, occlusal plane analyzer (Figs 1 and 2), consisted of a Fox Plane (Dr Frank Fox Dentsply/ York division, York, PA). Four long screws were attached at the four ends of occlusal plane relator arm. Care was taken to place these screws parallel to each other. Three metal plates (two in oblique direction and one in horizontal) were then attached to these screws. Two plates (oblique) were made parallel to occlusal plane relator arm. It served to analyze the parallelism of occlusal plane with ala-tragus line. The third plate (horizontal) was parallel to the frontal connecting arm which could relate the occlusal plane to interpupillary line. Metal collars were placed both above and below the plates on the screws. These helped in supporting the metal plates on the screws and to manage the movement of plates on the screws. A mark was made on both the sides of all the plates. Corresponding mark, which was in straight line to the mark on the plate, was placed on the arms of the Fox plane. These marks were made prominent by making an arrowhead, using tungstencarbide bur, on the plate. These marks helped in determining the parallelism of the plates by measuring the distance between the plates. A metal key was also provided with the instrument

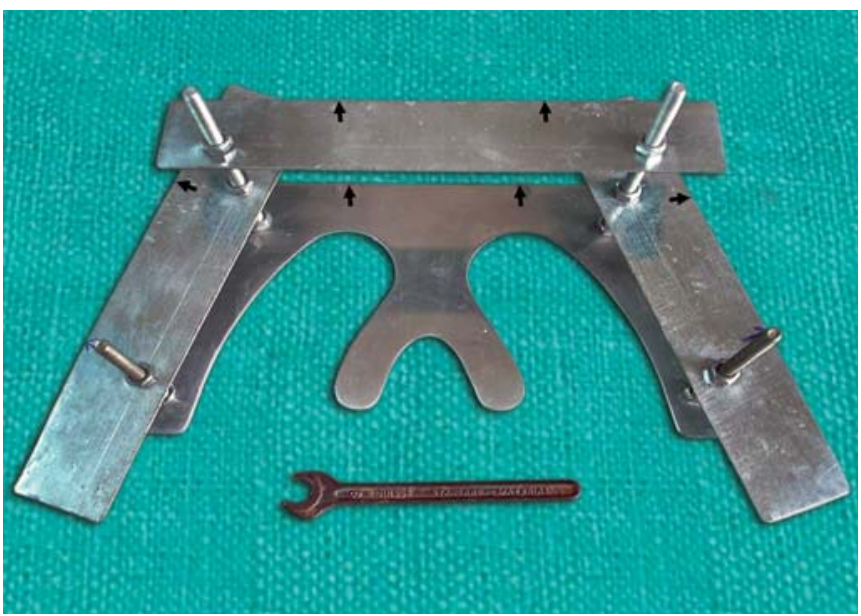

Fig. 1: Occlusal plane analyzer

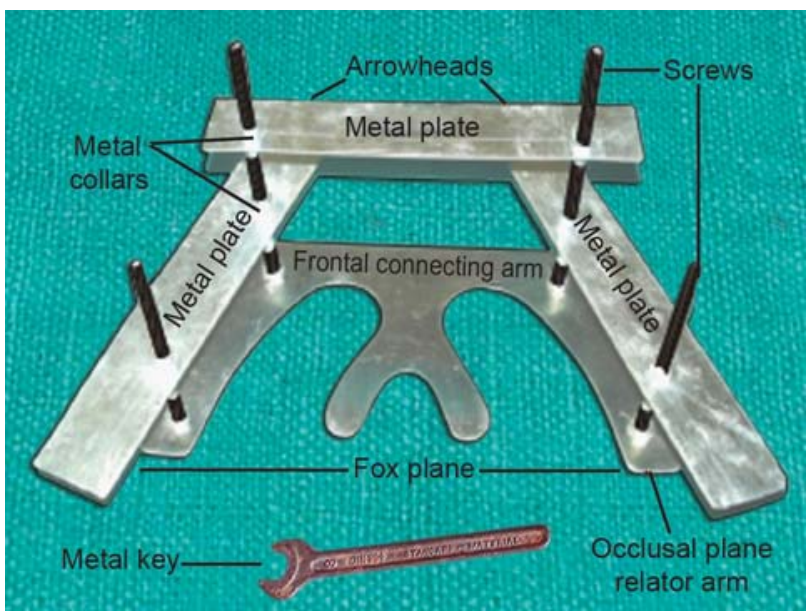

Fig. 2: Line diagram of the instrument to open the metal collars and help move the plates up and down. A metallic scale or vernier calipers can be used to check the distance between the two arrowheads on two parallel plates.

\section{PARALLELISM OF OCCLUSAL PLANE TO CAMPER'S LINE}

Patient is seated in an upright position in a dental chair. The occlusal plane analyzer is placed in the patient's mouth and held in position between the two occlusal rims (Fig. 3). Turns are given to the metal collars of the two screws supporting the side metal plates, thus opening the plate until it coincided with ala of the nose anteriorly and middle point on the tragus of the ear posteriorly.

The distance between the arrowheads on the two plates is then checked using metallic scale or vernier calipers (Fig. 4). This was repeated for the arrowheads on the other side of the same plate. Same distance between the two plates indicated parallel opening.

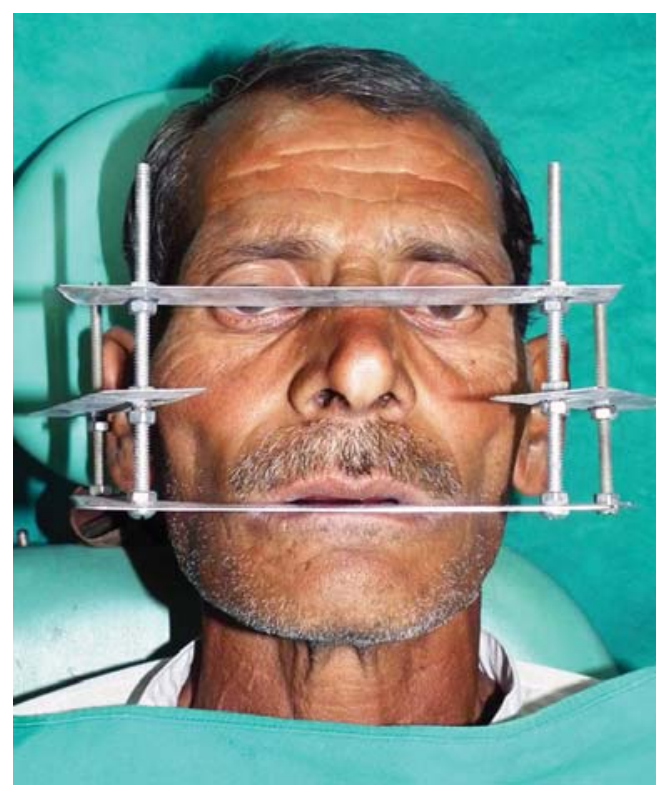

Fig. 3: Instrument in patient's mouth

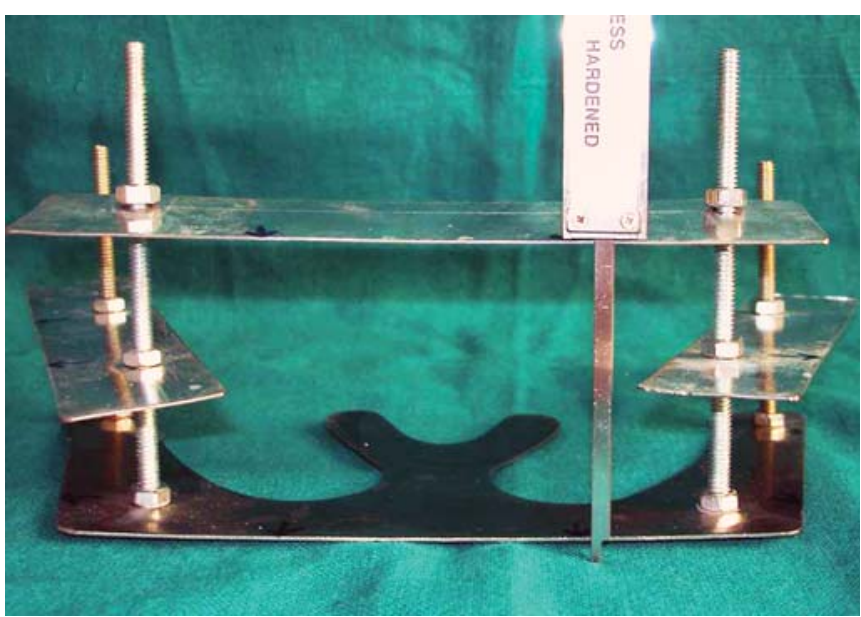

Fig. 4: Measuring the distance between two points using vernier calipers 
The occlusal rim is adjusted until same reading is obtained. Procedure is repeated for the other side also.

\section{PARALLELISM OF OCCLUSAL PLANE TO INTERPUPILLARY LINE}

The parallelism of occlusal plane with interpupillary line is checked using the same instrument. With the occlusal plane analyzer in the patient's mouth, the frontal metal plate is made parallel to the interpupillary line.

The parallelism is checked in the similar fashion.

\section{DISCUSSION}

The orientation of the occlusal plane, that is lost in patients rendered edentulous, should be relocated if complete dentures are to be esthetic and to function satisfactorily. Unfortunately, for the prosthetic educator, no accurate and practical means are presently available. Instead the student must learn by experience. Many methods have been proposed for determination of correct occlusal plane. Some of them have been offered as scientific, but as yet none is accurate. Others have been offered as helpful aids to good clinical judgement.

According to Zarb and Bolender, ${ }^{10}$ the lower rim is used to establish the occlusal plane. The corners of the mouth are marked on the occlusal rim to provide the anterior landmark for height of first premolars. Posteriorly the height is adjusted to two-third of the way-up of the retromolar pad. But practically, the corners of the mouth droop in most of the edentulous patients, which can lead to incorrect markings by the inexperienced students. Posteriorly, the retromolar pad was found to be the least reliable landmark for orienting the occlusal plane. ${ }^{11}$ Also, there is a controversy in literature regarding the exact point of location of the occlusal plane. Shigli K, Chetal BR and Jabade $\mathrm{J}^{12}$ (2005) said that the occlusal plane should be at the inferior 1/3rd of retromolar pad, while Yahia H Ismail and John F Bowman ${ }^{13}$ (1968) found it to be in middle third. Robert L Engelmeier ${ }^{14}$ (1996) and Donald F Reikie ${ }^{15}$ (2001) said that occlusal plane should lie at the junction of superior and middle 1/3rd of retromolar pad area while Corwin R Wright ${ }^{16}$ (2004) and Jack Piermatti ${ }^{17}$ (2006) said that it should be in superior 1/3rd.

According to Rahn and Heartwell, ${ }^{18}$ the occlusal rims are contoured so that the occluding surfaces of the maxillary rim are parallel with Camper's line. A tongue blade is placed on Camper's line and compared with Fox plane guide. In practice, a metallic scale is used along with Fox plane. The Fox plane is placed inside the mouth touching the occlusal rim and is held by the operator, while the metallic scale is held at the level of Camper's line or interpupillary line. In my experience, this procedure leads to errors in a number of cases as it is almost impossible to hold the scale steady. Also, the scale position changes every time the operator checks the parallelism. Moreover, when checking with naked eye, the operator has to be at eye-level to check the parallelism as any change in angulation of the eye leads to incorrect results. This, not only increases the chances of error but also make the procedure tedious and time-consuming.

A custom-made instrument for orientation of occlusal plane in completely edentulous patients has been described. This instrument had various advantages over the traditional method.

Accurate parallelism can be established as the parallelism is checked using scale or calipers, thus giving exact parallelism with definite values. There are reduced chances of error due to operator bias as the parallelism is not checked arbitrarily. Need to be at the eye level is eliminated and hence the chances of change in the angle of operator eye level leading to incorrect plane of occlusion are nil.

Simultaneous checking of parallelism of occlusal plane with Camper's line on both sides and interpupillary line helps in reducing chair time.

The instrument is simple and inexpensive. It is very easy to use and handle. The instrument can be held between the maxillary and mandibular occlusal rims by the patient, thus eliminating the need to constantly hold the instrument. The procedure can be carried out by a single user only.

The only disadvantage found included the bulk of the instrument and requirement of separate key to loosen the screws to allow movement of the plates.

\section{SUMMARY}

For the success of complete denture prosthesis, arranging the teeth in correct plane of occlusion is pivotal. Changes in the plane of occlusion modify the physical and functional relationship of the oral musculature leading to an alteration in function, comfort and also the esthetic value.

A custom-made instrument, occlusal plane analyzer, has been described to check the parallelism of the occlusal plane with the Camper's plane and the interpupillary plane.

But it must be constantly borne in mind that the occlusal plane is determined by the dynamics of function and not by any particular static relationship. It is up to the dentist's judgment and knowledge to choose the best possible level for the plane of occlusion.

\section{REFERENCES}

1. The glossary of prosthodontic terms. J Prosthet Dent 2005;94: 10-92.

2. Augsburger RH. Occlusal plane relation to facial type. J Prosthet Dent 1953;3:755-69.

3. Okane H, Yamashina T, Nagasawa T, Tsuru H. The effect of anteroposterior inclination of the occlusal plane on biting force. J Prosthet Dent 1979;42:497-501.

4. Rahn AO, Heartwell CM. Textbook of complete dentures (5th ed). Wolters Kluwer Co; 1986.

5. Rostamkhani F, Sahafian A, Kermani H. A cephalometric study on the relationship between the occlusal plane, ala-tragus and Camper's lines, in patients with Angle's class III Malocclusion. J Dent 2005;2:46-49. 
6. Scott JE. The Scott system of precision articulation in threedimensional occlusion. J Prosthet Dent May 1952;2(3): 362-79.

7. Javid NS. A technique for determination of the occlusal plane. J Prosthet Dent 1974 March;31(3):270-72.

8. Kazanoglu A, Unger JW. Determining the occlusal plane with the Camper's plane indicator. J Prosthet Dent April 1992; 67: 400-501.

9. Santana-Penin UA, Mora MJ. The occlusal plane indicator: A new device for determining the inclination of the occlusal plane. J Prosthet Dent 1998;80:374-75.

10. Zarb-Bolender. Prosthodontic treatment for edentulous patients-complete dentures and implant supported prosthesis (12th ed). Saunders Co, 2004.

11. Gupta R, Aeran H, Singh SP. Relationship of anatomic landmarks with occlusal plane. JIPS 2009;9(3):142-47.
12. Shigli K, Chetal BR, Jabade J. Validity of soft tissue landmarks in determining the occlusal plane. J Indian Prosthodont Soc 2005; 5:139-45.

13. Ismail YH, Bowman JF. Position of occlusal plane in natural and artificial teeth. J Prosthet Dent Nov 1968; 20(5):407-11.

14. Engelmeier RL. Complete denture esthetics. DCNA Jan 1996; 40(1):71-84.

15. Reikie DF. Orthodontically-assisted restorative dentistry. J Can Dent Assoc 2001;67(9):516-20.

16. Wright CR. Evaluation of the factors necessary to develop stability in mandibular dentures. J Prosthet Dent Dec 2004; 92(6): 509-18.

17. Piermatti J. Tooth position in full-mouth implant restorations: A case report. DART May-June 2006;209-13.

18. Rahn Arthur O, Heartwell Charles M. Textbook of complete dentures (5th ed). Wolters Kluwer Co, 1986. 